Original Research Paper

\title{
Medicinal Plants Used by Dayak Kanayatn Traditional Healers in Tonang Village Sengah Temila District Landak Regency
}

\author{
Rika Purnama Sari ${ }^{1}$, Fathul Yusro ${ }^{1}$, Yeni Mariani ${ }^{1 *}$ \\ ${ }^{1}$ Forestry Faculty, Tanjungpura University, Pontianak, Indonesia
}

\author{
Article History \\ Received : March 13 ${ }^{\text {th }}, 2021$ \\ Revised : April 15 ${ }^{\text {th }}, 2021$ \\ Accepted : April 21 th 2021 \\ Published : May 03 ${ }^{\text {th }}, 2021$ \\ *Corresponding Author: \\ Yeni Mariani, \\ Forestry Faculty, Tanjungpura \\ University, Pontianak \\ Email: \\ yeni.mariani81@gmail.com
}

\begin{abstract}
Plants have an essential role in people's lives because of their benefits and properties, such as for medicinal properties. In Landak District, only limited studies on medicinal plant had been carried out. This study analyzes the species of medicinal plants used by the Dayak Kanayatn tribal traditional healers in Tonang Village, Sengah Temila District, Landak Regency. The sampling used the snowball sampling technique. The results found that traditional healers of Dayak Kanayatn in Tonang Village used a total of 60 species belong to 36 families. The most utilized families $(8.47 \%)$ are Zingiberaceae and Asteraceae. The extensive use of plant habitus is herbs $(41,67 \%)$. Leaves are the most dominant plant part used $(44.78 \%)$, while the highest processing method was boiled $(39.74 \%)$. The highest form of use was drinking $(38.67 \%)$, the most common location of plants was in the yard $(67.74 \%)$, the highest plant status is cultivated $(52 \%)$, and the highest form of the potion is in the form of a mixture (72\%). The result shows that the traditional healers in the Dayak Kanayatn community in Tonang Village, Sengah Temila District, Landak Regency, still use medicinal plants to overcome health problems and treat disease.
\end{abstract}

Keywords: Dayak Kanayatn, medicinal plant, traditional healers, Tonang village

\section{Introduction}

Plants have an essential role in people's lives due to their benefits and properties, including for nutritious drugs. West Kalimantan has various types of plants used by the community since ancient times, especially by the Dayak tribe, the original tribe of West Kalimantan (Riadi et al. 2019). The traditional medication practicing in the Dayak tribe conducted by their traditional healers. One of the Dayak tribe that still use plants as traditional medicine is the Kanayatn. This tribe widely distributed in West Kalimantan especially in regencies of Mempawah, Bengkayang, Sanggau, Ketapang, and Landak (Rahmawati 2012).

Landak Regency is one of the area that is occupied by the Dayak Kanayatn tribe. In Landak District, several studies had been carried out related to the ethnobotany of medicinal plants. Ferdy et al. (2017) identified 44 species of medicinal plant (32 families) in the Village Ara wood, Menyuke District, Landak Regency. The Mingga et al. (2019), among the Dayak Ahe community in Raba Village, Menjalin District, Landak Regency, reported a total of 39 medicinal plants species from 30 families used this community. Other medicinal plant study in Kanayatn tribe were conducted by Fadillah $e t$ al. (2015) that obtained 29 species (20 families) of medicinal plants used by the Kanayatn Dayak tribe in Ambawang Village, Kubu District, Kubu Raya Regency. Efremila et al. (2015) also reported the medicinal plant study in Landak Regency, especially Kayu Tanam Village, Mandor district. Their study identified 50 species (32 families).

In Tonang Village, among the community, there are several traditional healers that are still practicing traditional medication. These traditional healers using the plant to treat diseases. However, there is no study on medicinal plant in this village. Therefore, it is necessary to conduct a research in order to 
document these traditional healer's knowledge of medicinal plants; thus, their experience is maintained. This study aims to analyze the types and levels of medicinal plants used by the Dayak Kanayatn traditional healers in Tonang Village, Sengah Temila District, Landak Regency.

\section{Materials and Method}

\section{Time and site of research}

This research had been conducted in Tonang village, Sengah Temila District, Landak Regency from June to August 2020. Starting from the data collection process, identifying medicinal plants species, and data analysis.

\section{Equipment}

The equipment used in this study are a list of the questioner for selected respondents, a camera for field documentation, a voice recorder to record the interview conversations, a computer, the medicinal plant identification books (Badrunasar and Santoso 2016; Baihaqi et al. 2017).

\section{Data Collection}

Data were collected by using interviews and observations. The respondents were selected using the snowball sampling technique. We started by interviewing the village head and elder community, thus leads us to Kanayatn traditional healers in their village. The selected traditional healers must fulfill the criteria like having medicinal plant knowledge and use it in their traditional medication.

\section{Data Analysis}

The data obtained were displayed in tables and graphics and analyzed descriptively. The data includes medicinal plant names (vernacular and scientific names), families, habitus, plant status, location, efficacy, plant part used, processing and usage method, duration of uses, and frequency of use.

\section{Result and Discussion}

In this study, a total of six traditional healers of Dayak Kanayatn in Tonang village were interviewed. They are classified into general traditional healers, birth assistants, and fracture specialists. In total, there are 60 species medicinal plant belong to 36 families used by these traditional healers. However, one species is not identified yet its scientific name (Table 1).

\section{Plant Utilization Based on Plant Family}

The traditional healers of Dayak Kanayatn in Tonang village use various species of medicinal plants in their medication. A total of 60 plant species (36 families) in different habitus are used in treating the diseases of the Dayak Kanayatn community in this village.

Zingiberaceae and Asteraceae's families are the most used by the traditional healer (Fig. 1). In many reports, we found that Zingiberaceae and Asteraceae are used in traditional medication of many communities (Yusro et al. 2020; Jadid et al. 2019; Rania et al. 2019; Nahdi et al. 2016.). These two families are the most widely used families in Asia, especially in the tropic region (Kumar et al., 2013).

Zingiberaceae and Asteraceae have long been known for their use as traditional medicine, and they are recorded in Ayurvedic medicine (Kumar et al., 2013). In traditional medication, various parts of Zingiberaceae's plant are used. Plants belonging to Zingiberaceae are known to possess active compounds and essential oils that benefit human life.

As the second famous family, Asteraceae is known for its biological activities (Michel et al. 2020). Several studies reported the active compound of this family, namely polyphenols, flavonoids, and diterpenoids (Koc et al. 2014).

\section{Plant Utilization Based on Plant Habitus}

There are several types of plants used by traditional healers of Dayak Kanayatn in Tonang village. The most extensive use is herbaceous $(41.67 \%)$, while the lowest is lianas $(3.33 \%)$ (Fig. 2). 
Rika Purnama Sari, et al. (2021). Jurnal Biologi Tropis, 21 (2): 324 - 335 DOI: http://dx.doi.org/10.29303/jbt.v21i2.2557

Tabel 1. The documented knowledge of medicinal plants used by traditional healers of Dayak Kanayatn in Tonang village

\begin{tabular}{|c|c|c|c|c|c|c|c|c|c|c|}
\hline No & Family & $\begin{array}{l}\text { Botanical } \\
\text { name }\end{array}$ & $\begin{array}{c}\text { Vernacular } \\
\text { Name }\end{array}$ & Indications & $\begin{array}{c}\text { Plant } \\
\text { part } \\
\text { usage }\end{array}$ & $\begin{array}{l}\text { Processing } \\
\text { Method }\end{array}$ & $\begin{array}{l}\text { Utilization } \\
\text { Method }\end{array}$ & Habitus & $\begin{array}{c}\text { Cultivated/ } \\
\text { Wild }\end{array}$ & $\begin{array}{c}\text { Plant } \\
\text { location }\end{array}$ \\
\hline 1 & Acanthaceae & $\begin{array}{l}\text { Clinacanthus } \\
\text { nutans } \mathrm{L}\end{array}$ & Kakamek & Broken bone & Leaves & Pounded & Patched & Shrub & Wild & Yard \\
\hline 2 & Acanthaceae & $\begin{array}{l}\text { Graptophylum } \\
\text { pictum } \mathrm{L}\end{array}$ & Lingkudikng & $\begin{array}{l}\text { Menstruation, } \\
\text { post- maternal } \\
\text { treatment, } \\
\text { fertilizing the } \\
\text { womb }\end{array}$ & $\begin{array}{l}\text { Leaves } \\
\text { and roots }\end{array}$ & Boiled & Drunk & Shrub & Cultivated & Yard \\
\hline 3 & Acanthaceae & $\begin{array}{l}\text { Justicia } \\
\text { gendarussa } \\
\text { Burm }\end{array}$ & $\begin{array}{l}\text { Tuba } \\
\text { lonyekng }\end{array}$ & $\begin{array}{l}\text { Broken bone and } \\
\text { inflammation }\end{array}$ & Leaves & $\begin{array}{l}\text { Pounded and } \\
\text { burnt }\end{array}$ & $\begin{array}{l}\text { Patched and } \\
\text { rubbed }\end{array}$ & Herb & Wild & Yard \\
\hline 4 & Alliaceae & $\begin{array}{l}\text { Allium sativum } \\
\mathrm{L}\end{array}$ & $\begin{array}{l}\text { Bawang } \\
\text { putih }\end{array}$ & $\begin{array}{l}\text { Sprain, } \\
\text { carbuncle, and } \\
\text { body pain }\end{array}$ & Bulbs & Pounded & Patched & Herb & Cultivated & Market \\
\hline 5 & Amaryllidaceae & $\begin{array}{l}\text { Crynum } \\
\text { asiaticum } \mathrm{L}\end{array}$ & $\begin{array}{l}\text { Bawang } \\
\text { bombai }\end{array}$ & Sprain & Bulbs & Pounded & Patched & Herb & Cultivated & Yard \\
\hline 6 & Amaranthaceae & Amaranthus sp. & Bayam & $\begin{array}{l}\text { Post-maternal } \\
\text { treatment }\end{array}$ & Leaves & Boiled & Eaten & Herb & Cultivated & Market \\
\hline 7 & Amaranthaceae & Celosia cristata & $\begin{array}{l}\text { Bunga } \\
\text { manok }\end{array}$ & Inflammation & Leaves & Pounded & Patched & Herb & Wild & Yard \\
\hline 8 & Annonaceae & $\begin{array}{l}\text { Annona } \\
\text { mucirata } \mathrm{L}\end{array}$ & $\begin{array}{l}\text { Nangka' } \\
\text { balanda }\end{array}$ & Fever, gastric & $\begin{array}{l}\text { Leaves } \\
\text { and roots }\end{array}$ & $\begin{array}{l}\text { Squeezed } \\
\text { and brewed }\end{array}$ & Drunk & Shrub & Cultivated & Yard \\
\hline 9 & Anisophylleaceae & $\begin{array}{l}\text { Anisophyllea } \\
\text { disticha }\end{array}$ & Sumiang & Diarrhea & Leaves & Direct used & Eaten & Shrub & Wild & Forest \\
\hline 10 & Apiaceae & $\begin{array}{l}\text { Coriandrum } \\
\text { sativum }\end{array}$ & Katumbar & Cholesterol & Fruits & Boiled & Drunk & Bush & Cultivated & Market \\
\hline 11 & Apiaceea & $\begin{array}{l}\text { Daucus carota } \\
\mathrm{L}\end{array}$ & Wortel & Eye treatment & Fruits & Shredded & Eaten & Bush & Cultivated & Market \\
\hline 12 & Araceae & $\begin{array}{l}\text { Colocasia } \\
\text { esculenta }\end{array}$ & Be'a & Wound & Leaves & Chopped & Patched & Herb & Wild & Yard \\
\hline 13 & Arecaceae & $\begin{array}{l}\text { Areca catachu } \\
\mathrm{L}\end{array}$ & Pinang & Broken bone & Midrib & Pounded & Patched & Tree & Cultivated & Yard \\
\hline 14 & Araliaceae & $\begin{array}{l}\text { Polyscias } \\
\text { scutellaria }\end{array}$ & $\begin{array}{l}\text { Kambang } \\
\text { mangkok }\end{array}$ & Inflammation & Leaves & $\begin{array}{l}\text { Pounded and } \\
\text { burnt }\end{array}$ & Rubbed & Bush & Cultivated & Yard \\
\hline
\end{tabular}


Rika Purnama Sari, et al. (2021). Jurnal Biologi Tropis, 21 (2): 324 - 335 DOI: http://dx.doi.org/10.29303/jbt.v21i2.2557

\begin{tabular}{|c|c|c|c|c|c|c|c|c|c|c|}
\hline No & Family & $\begin{array}{c}\text { Botanical } \\
\text { name }\end{array}$ & $\begin{array}{c}\text { Vernacular } \\
\text { Name }\end{array}$ & Indications & $\begin{array}{c}\text { Plant } \\
\text { part } \\
\text { usage }\end{array}$ & $\begin{array}{l}\text { Processing } \\
\text { Method }\end{array}$ & $\begin{array}{c}\text { Utilization } \\
\text { Method }\end{array}$ & Habitus & $\begin{array}{c}\text { Cultivated/ } \\
\text { Wild }\end{array}$ & $\begin{array}{c}\text { Plant } \\
\text { location }\end{array}$ \\
\hline 15 & Asteraceae & $\begin{array}{l}\text { Chromolaena } \\
\text { odorata } \mathrm{L}\end{array}$ & Carone & $\begin{array}{l}\text { Stomachache, } \\
\text { tonic, and fever }\end{array}$ & $\begin{array}{l}\text { Roots and } \\
\text { leaves }\end{array}$ & $\begin{array}{l}\text { Boiled, and } \\
\text { squeezed }\end{array}$ & $\begin{array}{l}\text { Drunk and } \\
\text { compressed }\end{array}$ & Shrub & Wild & Yard \\
\hline 16 & Asteraceae & $\begin{array}{l}\text { Elephantopus } \\
\text { scaber L }\end{array}$ & Jam teo & $\begin{array}{l}\text { Fever, } \\
\text { dysentery, } \\
\text { diarrhea }\end{array}$ & Leaves & Boiled & Drunk & Bush & Cultivated & Yard \\
\hline 17 & Asteraceae & $\begin{array}{l}\text { Gymnanthemum } \\
\text { amygdalinum }\end{array}$ & $\begin{array}{l}\text { Panyambung } \\
\text { nyawa }\end{array}$ & $\begin{array}{l}\text { Fever, } \\
\text { hypertension }\end{array}$ & Leaves & Boiled & $\begin{array}{l}\text { Compressed } \\
\text { and drunk }\end{array}$ & Shrub & Cultivated & Yard \\
\hline 18 & Asteraceae & $\begin{array}{l}\text { Blumea } \\
\text { balsamifera }\end{array}$ & Kimabo & $\begin{array}{l}\text { Post-maternal } \\
\text { treatment }\end{array}$ & Roots & Boiled & Drunk & Shrub & Wild & Forest \\
\hline 19 & Asteraceae & $\begin{array}{l}\text { Artemisia } \\
\text { vulgaris }\end{array}$ & Sasunge & $\begin{array}{l}\text { Post-maternal } \\
\text { treatment }\end{array}$ & Leaves & Boiled & Drunk & Herb & Cultivated & Yard \\
\hline 20 & Aspleniaceae & $\begin{array}{l}\text { Asplenium } \\
\text { nidus }\end{array}$ & Paku' sanah & $\begin{array}{l}\text { Broken bone, } \\
\text { cramp }\end{array}$ & Leaves & $\begin{array}{l}\text { Pounded and } \\
\text { boiled }\end{array}$ & $\begin{array}{l}\text { Patched and } \\
\text { eaten }\end{array}$ & Bush & Wild & Forest \\
\hline 21 & Blechnaceae & $\begin{array}{l}\text { Blechum } \\
\text { orientale }\end{array}$ & $\begin{array}{l}\text { Paku' } \\
\text { mamuraja }\end{array}$ & Carbuncle & Shoots & Pounded & Patched & Herb & Wild & Forest \\
\hline 22 & Crassulaceae & $\begin{array}{l}\text { Kalanchoe } \\
\text { pinnata }\end{array}$ & Padingin & Fever & Leaves & Boiled & Compressed & Herb & Cultivated & Yard \\
\hline 23 & Dilleniaceae & $\begin{array}{l}\text { Dillenia indica } \\
\mathrm{L}\end{array}$ & Abuatn & Inflammation & Leaves & $\begin{array}{l}\text { Pounded and } \\
\text { burnt }\end{array}$ & Rubbed & Shrub & Wild & Forest \\
\hline 24 & Euphorbiaceae & $\begin{array}{l}\text { Phyllanthus } \\
\text { urinaria } \mathrm{L}\end{array}$ & Antidur & $\begin{array}{l}\text { Fertilizing the } \\
\text { womb }\end{array}$ & Roots & Boiled & Drunk & Herb & Wild & Yard \\
\hline 25 & Euphorbiaceae & Macaranga sp. & Limpe'et & Inflammation & Leaves & $\begin{array}{l}\text { Pounded and } \\
\text { burnt }\end{array}$ & Rubbed & Shrub & Wild & Yard \\
\hline 26 & Euphorbiaceae & $\begin{array}{l}\text { Manihot } \\
\text { uttilisima Pohl }\end{array}$ & Ubi & Anemia & Leaves & Boiled & Eaten & Shrub & Cultivated & Yard \\
\hline 27 & Fabaceae & Vigna radiata & Kacambah & $\begin{array}{l}\text { Fertilizing the } \\
\text { womb }\end{array}$ & Shoots & Boiled & Eaten & Bush & Cultivated & Market \\
\hline 28 & Fabaceae & Cajanus cajan & Kacang bue & $\begin{array}{l}\text { Fertilizing the } \\
\text { womb }\end{array}$ & Roots & Boiled & Drunk & Herb & Cultivated & Yard \\
\hline 29 & Gleicheniaceae & $\begin{array}{l}\text { Gleichenia } \\
\text { linearis }\end{array}$ & Taboyo & Carbuncle & Shoots & Pounded & Patched & Bush & Wild & Forest \\
\hline 30 & Lamiaceae & $\begin{array}{l}\text { Plechtranthus } \\
\text { scutellarioides }\end{array}$ & Ati-ati & Gastric & Leaves & Brewed & Drunk & Herb & Cultivated & Yard \\
\hline
\end{tabular}


Rika Purnama Sari, et al. (2021). Jurnal Biologi Tropis, 21 (2): 324 - 335 DOI: http://dx.doi.org/10.29303/jbt.v21i2.2557

\begin{tabular}{|c|c|c|c|c|c|c|c|c|c|c|}
\hline No & Family & $\begin{array}{l}\text { Botanical } \\
\text { name }\end{array}$ & $\begin{array}{l}\text { Vernacular } \\
\text { Name }\end{array}$ & Indications & $\begin{array}{c}\text { Plant } \\
\text { part } \\
\text { usage }\end{array}$ & $\begin{array}{l}\text { Processing } \\
\text { Method }\end{array}$ & $\begin{array}{l}\text { Utilization } \\
\text { Method }\end{array}$ & Habitus & $\begin{array}{c}\text { Cultivated/ } \\
\text { Wild }\end{array}$ & $\begin{array}{c}\text { Plant } \\
\text { location }\end{array}$ \\
\hline 31 & Liliaceae & $\begin{array}{l}\text { Eleutherine } \\
\text { americana Merr }\end{array}$ & $\begin{array}{l}\text { Bawang } \\
\text { lama }\end{array}$ & $\begin{array}{l}\text { Breast cancer, } \\
\text { carbuncle, and } \\
\text { hypertension }\end{array}$ & Bulbs & $\begin{array}{l}\text { Pounded and } \\
\text { Boiled }\end{array}$ & $\begin{array}{l}\text { Patched and } \\
\text { drunk }\end{array}$ & Herb & Cultivated & Yard \\
\hline 32 & Loranthaceae & $\begin{array}{l}\text { Scurrula } \\
\text { atropurpurea }\end{array}$ & Korouncit & Broken bone & Roots & Pounded & Patched & Shrub & Wild & Yard \\
\hline 33 & Lomariopsidaceae & $\begin{array}{l}\text { Nephrolepis } \\
\text { biserrata }\end{array}$ & Paku' uban & Remove scars & Shoots & Pounded & Patched & Herb & Wild & Yard \\
\hline 34 & Malvaceae & $\begin{array}{l}\text { Abelmoschus } \\
\text { esculentus } \mathrm{L}\end{array}$ & $\begin{array}{l}\text { Kacang } \\
\text { tanuk }\end{array}$ & Constipation & Fruits & Boiled & Eaten & Bush & Cultivated & Yard \\
\hline 35 & Malvaceae & $\begin{array}{l}\text { Hibiscus rosa- } \\
\text { sinensis L }\end{array}$ & $\begin{array}{l}\text { Kembang } \\
\text { sepatu }\end{array}$ & Carbuncle & Leaves & Pounded & Patched & Shrub & Cultivated & Yard \\
\hline 36 & Melastomaceae & $\begin{array}{l}\text { Melastoma } \\
\text { candidum }\end{array}$ & Lingkodok & Diarrhea & Leaves & $\begin{array}{l}\text { Direct used } \\
\text { and boiled }\end{array}$ & $\begin{array}{l}\text { Eaten and } \\
\text { drunk }\end{array}$ & Herb & Wild & Yard \\
\hline 37 & Meliaceae & $\begin{array}{l}\text { Melia } \\
\text { azedarach }\end{array}$ & Bambali & Broken bone & Stem & Direct used & Patched & Tree & Wild & Forest \\
\hline 38 & Myrtaceea & $\begin{array}{l}\text { Psidium } \\
\text { guajava L }\end{array}$ & Jamu karas & Diarrhea & Leaves & Boiled & Drunk & Shrub & Wild & Yard \\
\hline 39 & Myrtaceae & $\begin{array}{l}\text { Syzygium } \\
\text { polyanthum }\end{array}$ & Salam & $\begin{array}{l}\text { Cholesterol, } \\
\text { hypertension }\end{array}$ & $\begin{array}{l}\text { Leaves } \\
\text { and roots }\end{array}$ & Boiled & Drunk & Shrub & Cultivated & Yard \\
\hline 40 & Myrtaceae & $\begin{array}{l}\text { Syzygium } \\
\text { zeylanicum }\end{array}$ & Ubah & $\begin{array}{l}\text { Breast cancer, } \\
\text { carbuncle }\end{array}$ & Roots & Pounded & Patched & Shrub & Wild & Forest \\
\hline 41 & Orchidaceae & $\begin{array}{l}\text { Phalaenopsis } \\
\text { amabilis }\end{array}$ & $\begin{array}{l}\text { Anggrek } \\
\text { putih }\end{array}$ & Smallpox & Leaves & Pounded & Patched & Bush & Wild & Forest \\
\hline 42 & Palmaceae & Cocos nucifera & Kalapa & Gastric & Roots & Brewed & Drunk & Tree & Cultivated & Yard \\
\hline 43 & Piperaceae & Piper bettle L & Karakek & $\begin{array}{l}\text { Vaginal } \\
\text { discharge, } \\
\text { cough, allergy }\end{array}$ & Leaves & $\begin{array}{l}\text { Boiled and } \\
\text { burnt }\end{array}$ & $\begin{array}{l}\text { Bath, drunk } \\
\text { and rubbed }\end{array}$ & Liana & Wild & Yard \\
\hline 44 & Piperaceae & Piper nigrum L & Sahakng & $\begin{array}{l}\text { Post-maternal } \\
\text { treatment }\end{array}$ & Fruits & Boiled & Drunk & Liana & Cultivated & Garden \\
\hline 45 & Poaceae & $\begin{array}{l}\text { Bambusa } \\
\text { vulgaris } \mathrm{S}\end{array}$ & Buluh bala & Lumbago & $\begin{array}{l}\text { Roots and } \\
\text { midrib }\end{array}$ & Boiled & Drunk & Shrub & Wild & Forest \\
\hline 46 & Poaceae & $\begin{array}{l}\text { Imperata } \\
\text { cylindrica } \mathrm{L}\end{array}$ & Padakng & $\begin{array}{l}\text { Fertilizing the } \\
\text { womb }\end{array}$ & Roots & Boiled & Drunk & Herb & Wild & Yard \\
\hline 47 & Poaceae & $\begin{array}{l}\text { Coix lacryma- } \\
\text { Jobi }\end{array}$ & Anyalik & Breast cancer & Roots & Pounded & Patched & Herb & Wild & Yard \\
\hline
\end{tabular}


Rika Purnama Sari, et al. (2021). Jurnal Biologi Tropis, 21 (2): 324 - 335 DOI: http://dx.doi.org/10.29303/jbt.v21i2.2557

\begin{tabular}{|c|c|c|c|c|c|c|c|c|c|c|}
\hline No & Family & $\begin{array}{c}\text { Botanical } \\
\text { name }\end{array}$ & $\begin{array}{c}\text { Vernacular } \\
\text { Name }\end{array}$ & Indications & $\begin{array}{c}\text { Plant } \\
\text { part } \\
\text { usage }\end{array}$ & $\begin{array}{c}\text { Processing } \\
\text { Method }\end{array}$ & $\begin{array}{c}\text { Utilization } \\
\text { Method }\end{array}$ & Habitus & $\begin{array}{c}\text { Cultivated/ } \\
\text { Wild }\end{array}$ & $\begin{array}{c}\text { Plant } \\
\text { location }\end{array}$ \\
\hline 48 & Poaceae & $\begin{array}{l}\text { Cymbopogon } \\
\text { citratus }\end{array}$ & Sare & $\begin{array}{l}\text { Flatulence, } \\
\text { tonic, } \\
\text { cholesterol }\end{array}$ & Stem & Boiled & Drunk & Herb & Cultivated & Yard \\
\hline 49 & Rutaceae & $\begin{array}{l}\text { Citrus } \\
\text { amblycarpa }\end{array}$ & $\begin{array}{l}\text { Limo } \\
\text { sambal }\end{array}$ & Carbuncle & Leaves & Pounded & Patched & Shrub & Cultivated & Yard \\
\hline 50 & Salicaceae & $\begin{array}{l}\text { Flacourtia } \\
\text { rukam }\end{array}$ & Rukapm & $\begin{array}{l}\text { Carbuncle, } \\
\text { hypertension }\end{array}$ & $\begin{array}{l}\text { Leaves } \\
\text { and Roots }\end{array}$ & $\begin{array}{l}\text { Direct used } \\
\text { and boiled }\end{array}$ & $\begin{array}{l}\text { Eaten and } \\
\text { Drunk }\end{array}$ & Tree & Wild & Forest \\
\hline 51 & Sapindaceae & $\begin{array}{l}\text { Nephelium } \\
\text { lappaceum } \mathrm{L}\end{array}$ & Rambutan & Gastric & Roots & Brew & Drunk & Tree & Cultivated & Yard \\
\hline 52 & Solanaceae & $\begin{array}{l}\text { Physalis } \\
\text { angulata } \mathrm{L}\end{array}$ & Lalatup & $\begin{array}{l}\text { Dysentery, } \\
\text { Gastric, } \\
\text { hypertension }\end{array}$ & Roots & $\begin{array}{l}\text { Boiled and } \\
\text { brewed }\end{array}$ & Drunk & Herb & Wild & Yard \\
\hline 53 & Solanaceae & $\begin{array}{l}\text { Solanum } \\
\text { torvum }\end{array}$ & Marajakng & Dental treatment & Roots & Boiled & Drunk & Shrub & Wild & Yard \\
\hline 54 & Verbenaceae & Vitex pinnata & Laban & Gastric & Leaves & $\begin{array}{l}\text { Direct used, } \\
\text { boiled, and } \\
\text { brewed }\end{array}$ & $\begin{array}{l}\text { Eaten and } \\
\text { Drunk }\end{array}$ & Tree & Wild & Forest \\
\hline 55 & Zingiberaceae & $\begin{array}{l}\text { Boesenbergia } \\
\text { pandurata }\end{array}$ & $\begin{array}{l}\text { Antamu } \\
\text { kunci }\end{array}$ & $\begin{array}{l}\text { Sprain, body } \\
\text { pain }\end{array}$ & Rhizomes & Pounded & Patched & Herb & Cultivated & Yard \\
\hline 56 & Zingiberaceae & $\begin{array}{l}\text { Curcuma } \\
\text { domestica }\end{array}$ & Unyit & $\begin{array}{l}\text { Breast cancer, } \\
\text { vaginal } \\
\text { discharge }\end{array}$ & Rhizomes & $\begin{array}{l}\text { Pounded and } \\
\text { boiled }\end{array}$ & $\begin{array}{l}\text { Patched and } \\
\text { drunk }\end{array}$ & Herb & Cultivated & Yard \\
\hline 57 & Zingiberaceae & $\begin{array}{l}\text { Curcuma } \\
\text { zanthorrhiza } \mathrm{R}\end{array}$ & Temulawak & Breast cancer & Rhizomes & Pounded & Patched & Herb & Cultivated & Yard \\
\hline 58 & Zingiberaceae & $\begin{array}{l}\text { Kaemferia } \\
\text { galanga } \mathrm{L}\end{array}$ & Cakur & $\begin{array}{l}\text { Fertilizing the } \\
\text { womb, post- } \\
\text { maternal } \\
\text { treatment }\end{array}$ & $\begin{array}{l}\text { Rhizomes } \\
\text { and leaves }\end{array}$ & Boiled & Drunk & Herb & Cultivated & Yard \\
\hline 59 & Zingiberaceae & $\begin{array}{l}\text { Zingiber } \\
\text { officinale Linn }\end{array}$ & $\begin{array}{l}\text { Lahia’ } \\
\text { merah }\end{array}$ & $\begin{array}{l}\text { Inflammation, } \\
\text { Sprain, body } \\
\text { pain, broken } \\
\text { bone, post- } \\
\text { maternal } \\
\text { treatment, and } \\
\text { tonic }\end{array}$ & Rhizomes & $\begin{array}{l}\text { Pounded, } \\
\text { burnt and } \\
\text { boiled }\end{array}$ & $\begin{array}{l}\text { Patched, } \\
\text { rubbed, and } \\
\text { drunk }\end{array}$ & Herb & Cultivated & Yard \\
\hline
\end{tabular}


Rika Purnama Sari, et al. (2021). Jurnal Biologi Tropis, 21 (2): 324 - 335 DOI: http://dx.doi.org/10.29303/jbt.v21i2.2557

\begin{tabular}{|c|c|c|c|c|c|c|c|c|c|c|}
\hline No & Family & $\begin{array}{c}\text { Botanical } \\
\text { name }\end{array}$ & $\begin{array}{c}\text { Vernacular } \\
\text { Name }\end{array}$ & Indications & $\begin{array}{c}\text { Plant } \\
\text { part } \\
\text { usage }\end{array}$ & $\begin{array}{c}\text { Processing } \\
\text { Method }\end{array}$ & $\begin{array}{c}\text { Utilization } \\
\text { Method }\end{array}$ & Habitus & $\begin{array}{c}\text { Cultivated/ } \\
\text { Wild }\end{array}$ & $\begin{array}{c}\text { Plant } \\
\text { location }\end{array}$ \\
\hline 60 & - & - & Tiba'akng & $\begin{array}{l}\text { Inflammation } \\
\text { dan diarrhea }\end{array}$ & Leaves & $\begin{array}{l}\text { Pounded and } \\
\text { direct used }\end{array}$ & $\begin{array}{l}\text { Patched and } \\
\text { eaten }\end{array}$ & Tree & Wild & Forest \\
\hline
\end{tabular}




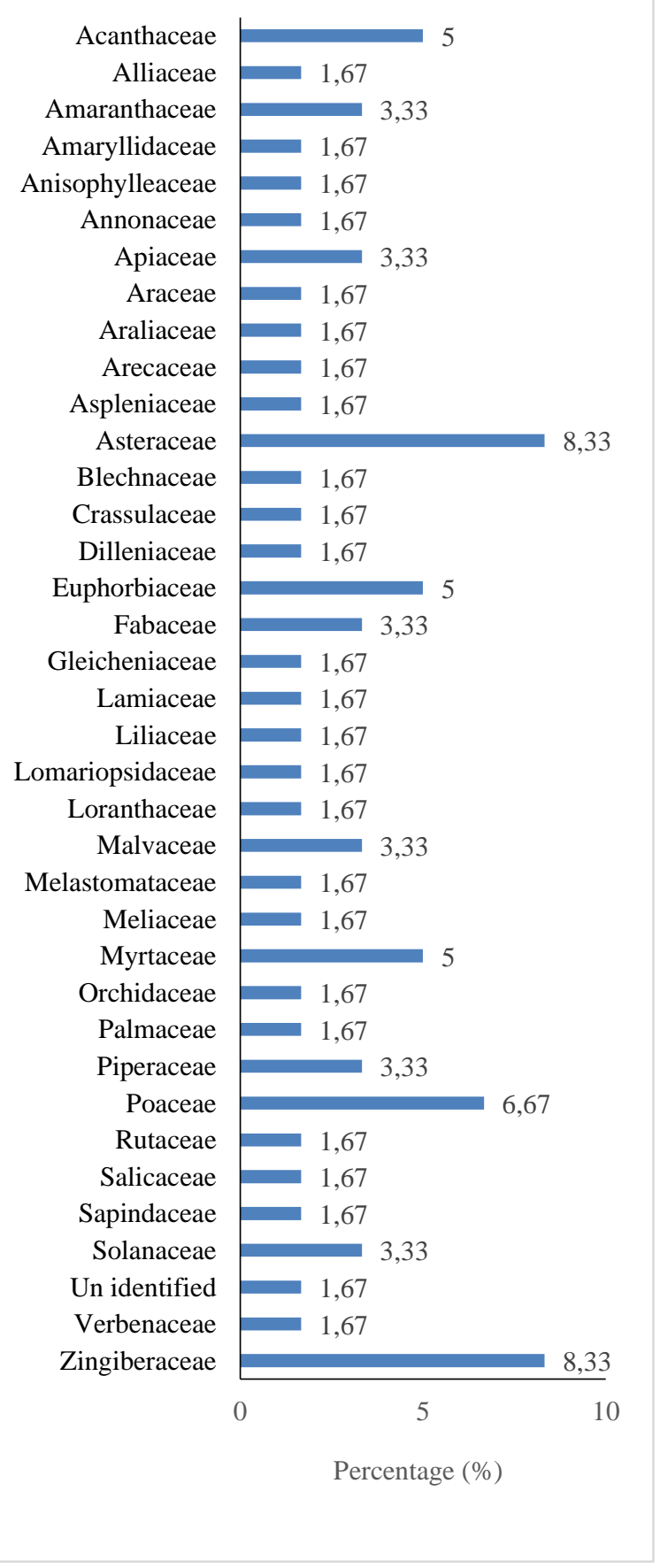

Figure 1. The Percentage of plant family used by traditional healers of Dayak Kanayatn in Tonang village

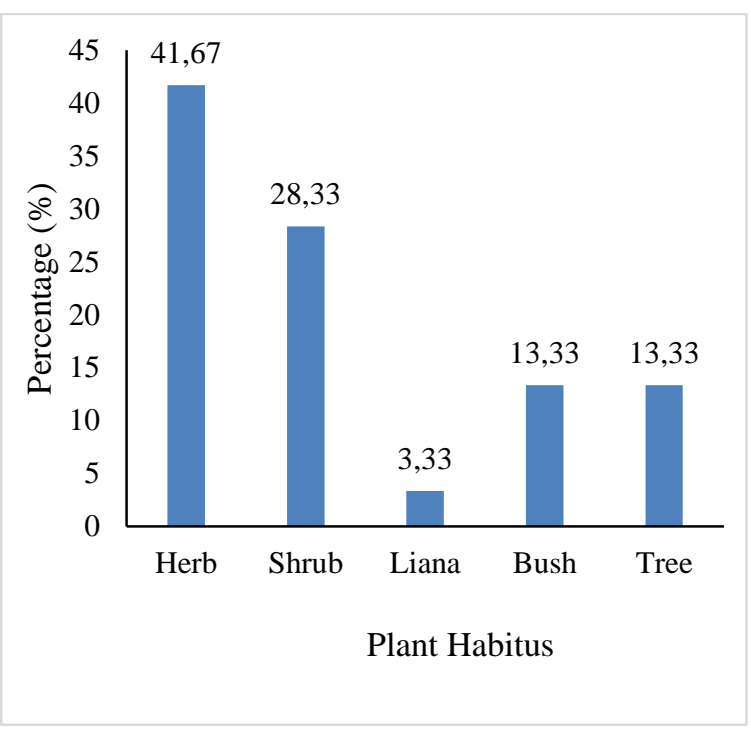

Figure 2. The percentage of plants habitus used by traditional healers of Dayak Kanayatn in Tonang village

Yusro et al. (2020) also reported that herb is the main habitus of the medicinal plant used by traditional healers in Merpak and Kebong villages. The extensive use of herb because they are commonly more found and grown in the yard, garden, and forest. The study of Mussarat et al. (2014) in the Indus River, Pakistan also showed that herb was commonly used for medicinal plant. Also, these plants contain many bioactive compounds; therefore, the local healer in this region utilizes them in many traditional remedies.

\section{Medicinal Plant Part in Traditional Medicinal Treatment}

The traditional healers of Dayak Kanayatn in Tonang village use various medicinal plant parts, namely rhizome, roots, fruit, leaves, midrib, shoots, and bulb. Figure 3 present the percentage of these plant part. 


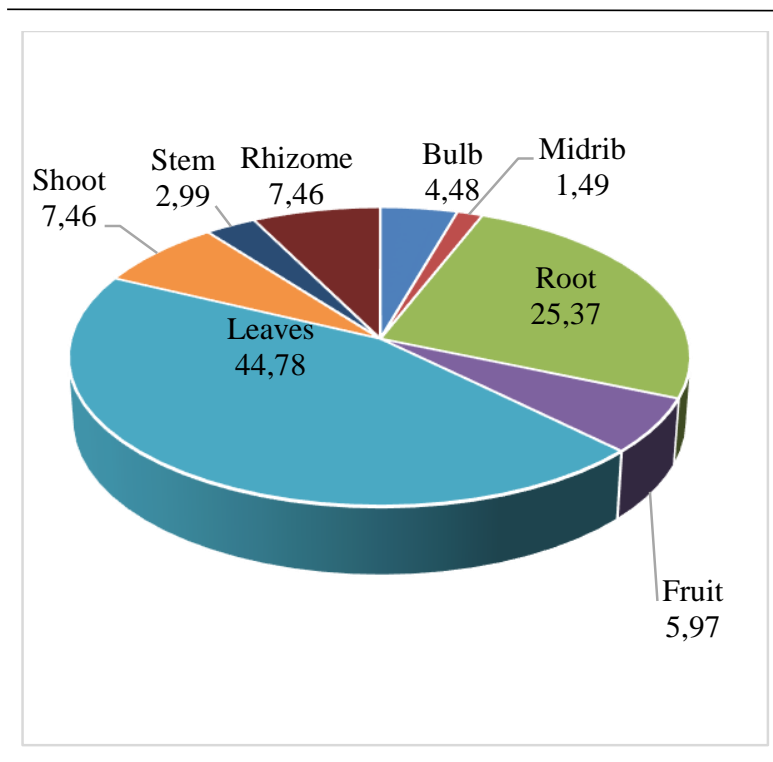

Figure 3. The percentage of plants part used by traditional healers of Dayak Kanayatn in Tonang village

In this study, we found that the highest use of plant part is the leaves. This study supported with the report of Yusro et al. (2020). In many studies, it was reported that leaves are used as a potion to treats diseases. In this village, the traditional healers of Dayak Kanayatn use leaves to treat ailments, bone fracture treatment, and post-maternal treatment.

Samoisy and Mahomoodally (2015) also reported the same result in the Island of Rodrigues of the Republic of Mauritius and Kadir et al. (2020) in the Chittagong Hills of Bangladesh. The leaves were almost widely used by people in those areas as medicinal ingredients. Leaves, one of the plant organs where photosynthetic activities take place, contain various active ingredients that can be used as medicine (Ullah et al. 2020).

\section{Medicinal Plant Processing Method in Traditional Medicinal Treatment}

We interviewed the traditional healers of Dayak Kanayatn in Tonang village about preparing the medicinal plants potion to treat the disease. The traditional healers in this village use several ways to prepare the medicinal plant potion (Fig. 4).

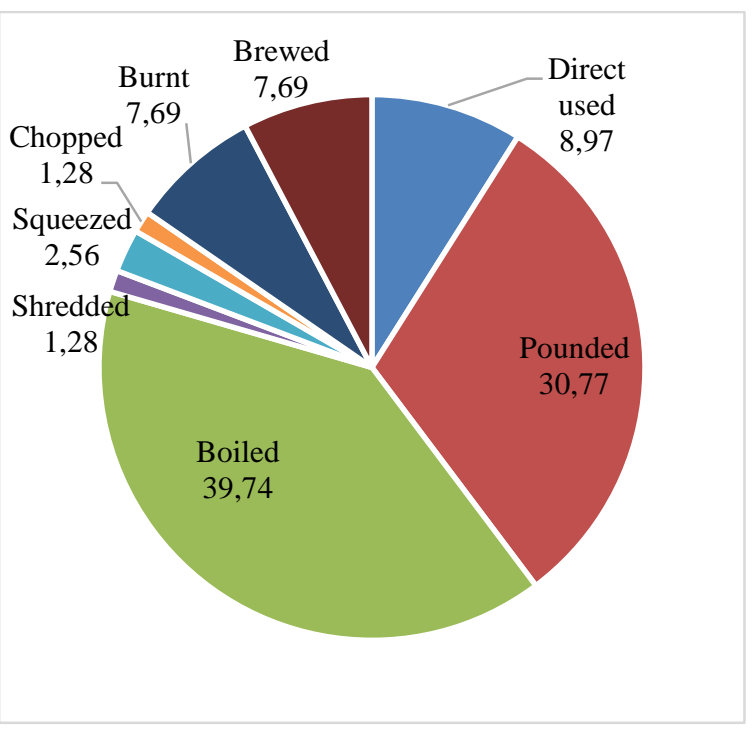

Figure 4. The percentage of processing method of medicinal plant used by traditional healers of Dayak Kanayatn in Tonang village

The preparation method they used is boiled, pounded, chopped, squeezed, shredded, burnt, and brewed. Some medicinal plants also directly (direct used). According to these traditional healers, most of the medicinal plant potion is made by boiling the plant $(39,74 \%)$. Our previous report on traditional healers in Merpak and Kebong villages also found a similar result (Yusro et al. 2020).

In this study, most of the healers prepared the medicinal plant by boiling plant part because they believed it will have to have biological properties with a water solvent. This preparation method is similar to the practiced of traditional healers in the Indus river and Algeria (Mussarat et al. 2014; Taibi et al. 2020). When the medicinal plants are boiled, their active substances may quickly dilute. They believe that the patient will be cured soon after they drink the potion.

\section{Medicinal Plant Utilization Method in Traditional Medicinal Treatment}

In this study, we asked the traditional healers of Dayak Kanayatn their utilization method of medicinal plant potion. According to them, there are several methods in utilization the medicinal plant potion. These methods are 
patched, rubbed, eaten, drunk, compressed, and bathed (Fig. 5).

The suggestion of the utilization method of the potion depends on the type of disease. Usually, a patient with an internal illness will be given the medicinal plant potion with the oral administration method, which can be eaten or drink. In this village, most medicinal plant potion is administered with drunk (38.67\%) and patched (32\%) (Fig. 5).

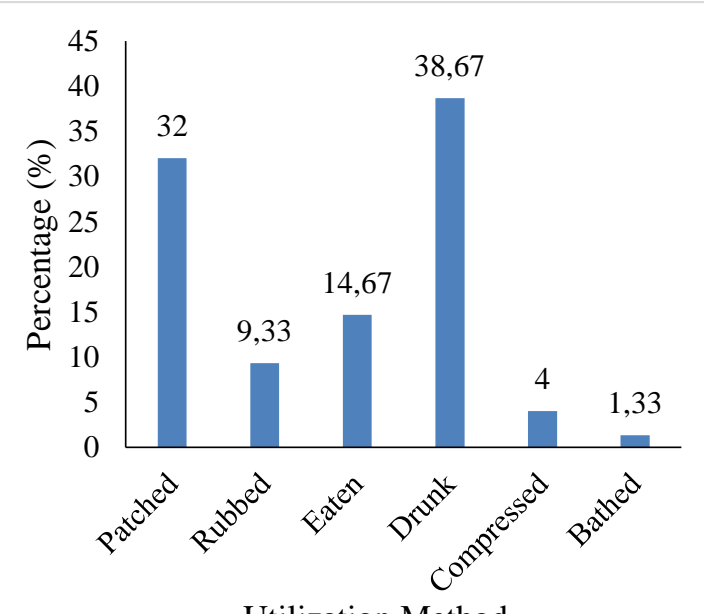

Utilization Method

Figure 5. The percentage of utilization method of medicinal plant used by traditional healers of Dayak Kanayatn in Tonang village

This result resonance with our previous result in Merpak and Kebong villages, and traditional midwife in Kayong Utara, where the traditional medication practices by the traditional healers in those villages also prescribe the same administration method of medicinal plant potion to their patient (Yusro et al. 2020; Rania et al. 2019).

\section{Medicinal Plant Harvested Location Used in Traditional Medicinal Treatment}

Based on the interviews, we found a total of 4 locations the medicinal plants harvested, namely forest, garden, market, and yard (Fig. 6). In traditional medication, the healers prepare the medicinal potions. Some of the medicinal plants are taken from the forest near their village, in their garden, and in the yard. Among these plants also can be find in the market near their village.

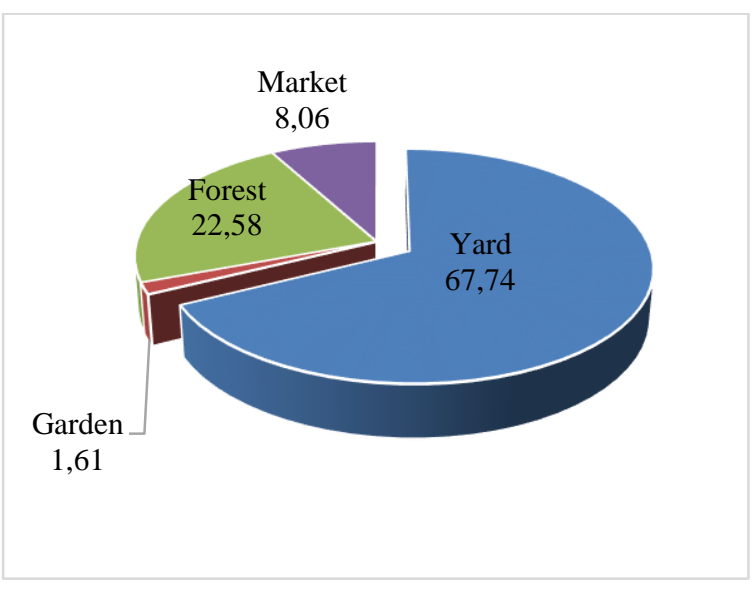

Figure 6. The percentage of harvested location of medicinal plant used by traditional healers of Dayak Kanayatn in Tonang village

The yard is the primary site where the traditional healers take the medicinal plant and use it in their conventional medication (67.74\%). We assumed that this village's traditional healers already cultivate their medicinal plant used in their regular medication. Thus, they will able to harvest it quickly when needed. Although the yard is the primary site where they take the medicinal plant, the traditional healers also take plants from the forest for their remedies $(22.58 \%)$.

\section{Medicinal Plant Cultivation Status}

Figure 7 shows that the traditional healer's plant in Tonang village are cultivated (52\%). While the percentage of wild species is slightly lower than cultivated species.

During the interviews, the traditional healers explains that they cultivate the plants in the yard and in the garden. Therefore, they will be easy to take it if they need. Although most of their plants are cultivated, they still used the wild ones. Some of the plants also live in the wild. We assumed these plants are live in the forest or plant that easy to grow, thus they not yet try to cultivate it. 


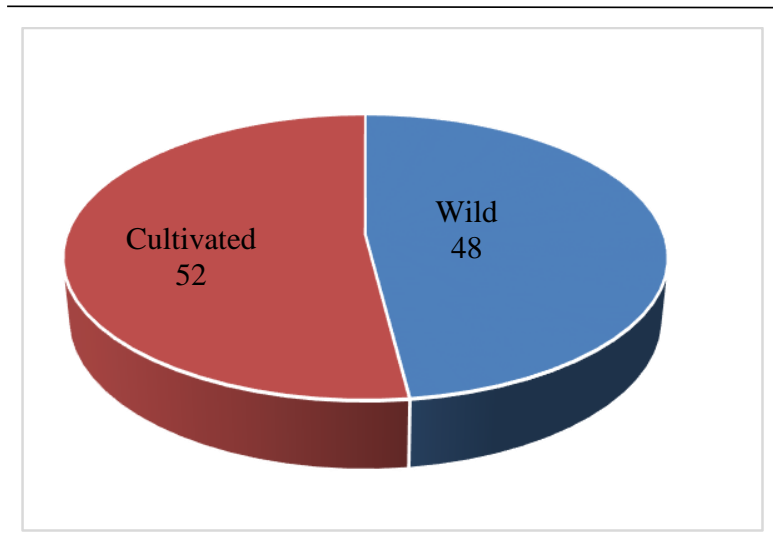

Figure 7. The percentage of medicinal plant cultivation status used by traditional healers of Dayak Kanayatn in Tonang village

\section{Conclusion}

In the Dayak community of Tonang Village, there are still traditional healers who practice conventional medication with the medicinal plant. The community uses this medication to treat various diseases and healthcare. It shows that the Dayak Kanayatn traditional knowledge on medicinal plants still maintained. They also conduct an effort to cultivate the plants used in their medication; thus, it prevents extinction.

\section{Acknowledgement}

We acknowledge the people of Tonang Village Sengah Temila District, Landak Regency, especially the traditional healers who have helped in this study.

\section{References}

Badrunasar, A., \& Santoso H.B. (2016). Tumbuhan Liar Berkhasiat Obat. Forda Press. Jakarta.

Baihaqi, A., Khoir, M., Satrio, MB., Sudrajat, A.K., Rachmam N.P., Nazhat, A., Khanzaa, S., Nunung, N., Duryat L.D. Hadanursamsi ,R. \& Nurjaman J. (2017). Tumbuhan Obat \& Satwa Liar. KEHATI. Jakarta.

Efemilia., Wardenaar, E. \& Sisillia, L. (2015).
Studi Ethnobotani Tumbuhan Obat Oleh Etnis Suku Dayak di Desa Kayu Tanam Kecamatan Mandor Kabupaten Landak. Jurnal Hutan Lestari. 3(2): 234-246. DOI: http://dx.doi.org/10.26418/jhl.v3i2. 10310.

Fadillah., Lovadi I. \& Linad, R. (2015). Pemanfaatan Tumbuhan Obat dalam Pengobatan Tradisional Masyarakat Suku Dayak Kanayatn di Desa Ambawang Kecamatan Kubu Raya. Jurnal Protobiont. 4(3): 49-59. DOI: http://dx.doi.org/10.26418/protobiont.v4i 3.13303 .

Ferdy, R., Usman, F.H. \& Sisillia, L. (2017). Pemanfaatan Tumbuhan Obat oleh Masyarakat Desa Kayu Ara di Kecamatan Manyuke Kabupaten Landak. Jurnal Hutan Lestari. 5(2): 452-459. DOI: http://dx.doi.org/10.26418/jhl.v5i2. 20280.

Jadid, N., Kurniawan, E., Himayani, C.E.S., Andriyani., Prasetyowati I., Purwani K.I., Muslihatin, W., Hidayati, D.\& Tjahjaningrum I.T.D. (2019). An Ethobotanical Study of Medicinal Plants Used by Tengger Tribe in Ngadisari Village, Indonesia. PLoS ONE. 15(7): e0235886. http://doi.org/10.1371/journal.pone.02358 86.

Kadir, M.F., Karmoker, J.R., Rashedul-Alam, Md., Jahan S.R., Mahbub, S. \& Mia, M.M.K. (2015). Ethnopharmacological Survey of Medicinal Plants Used by Traditional Healers and Indigenous People in Chittagong Hill Tracts, Bangladesh, For the Treatment of Snakebite. 2015.i. http://dx.doi.org/10.1155/2015/871675.

Koc, S., Isgor, B.S., Isgor, Y.G., Moghaddam, N.S. \& Yildrim, O. (2014). The Potential Medicinal Value of Plant From Asteraceae Family With Antioxidant Defense Enzymes as Biological Targets. Pharmaceutical Biology. 53(5): 746-751. DOI: http://dx.doi.org/10.3109/13880209. 2014.942788 
Michel, J., Abd Rani. \& Husain, K. (2020). A Review on The Potential Use of Medicinal Plants From Asteraceae and Lamiaceae Plant Family in Cardiovascular Diseases. Frontiers in Pharmacology. 11: 852. DOI: http://dx.doi.org/10.3389/fphar.2020.0085 2

Kumar, K.M., Asish, G.R., Sabu, M. \& Balachandran, I. (2013). Significance of Gingers (Zingiberaceae) in Indian System of Medicine-Ayurveda: An Overview. Ancient Science of Life. 32(4): 253-261. DOI: http://dx.doi.org/10.4103/02577941.131989

Mingg,a M., Oramahi, H.A., \& Tavita, G.E. (2019). Pemanfaatan Tumbuhan Obat oleh Masyarakat di Desa Raba Kecamatan Menjalin Kabupaten. Jurnal Hutan Lestari 7(1): $\quad 97 \quad-\quad 105$. DOI: http://dx.doi.org/10.26418/jhl.v7i1. 31005.

Mussarat, S., AbdlEl-Salam, N.M., Tariq, A., Wazir, S.M., Ullah, R. \& Adnan, M. (2014). Use of Ethnomedicinal Plants by The Pople Living Around Indus River. Evidence-Based Complementary and Alternative Medicine. 2014.i. DOI: http://dx.doi.org/10.1155/2014/212634.

Nahdi, M.S., Martiwi, I.N.A. \& Arsyah, D.C. (2016). The Ethnobotany of Medicinal Plants in Suporting The Family Health in Turgo, Yogyakarta. Indonesia. Biodiversitas. http://dx.doi.org/10.13057/biodiv/d17026 8.

Rahmawati, N.P.N. (2012). Nilai Pendidikan dalam Budaya Menanam Padi Suku Dayak Kanayatn di Kalimantan Barat. Patanjala . Jurnal Penelitian Sejarah dan Budaya 4 (1): $\quad 15-25$. DOI: http://dx.doi.org/ 10.30959/patanjala.v4i1 .121

Rania, Yusro, F., Wardenaar, E., \& Mariani, Y. (2019). Studi Pemanfaatan Tumbuhan Obat oleh Pengobat Tradisional untuk
Mengatasi Masalah Kewanitaan di Desa Masbangun Kecamatan Teluk Batang Kabupaten Kayong Utara. Jurnal Borneo Akcaya, 5(2), 84-94. http://jurnallitbang.kalbarprov.go.id:8088/index.php/l itbang/article/view/113

Riadi, R., Oramahi, H.A. \& Yusro, F. (2019). Pemanfaatan Tumbuhan Obat oleh Suku Dayak Kanayatn di Desa Mamek Kecamatan Menyuku Kabupaten Landak. Jurnal Hutan Lestari. 7(2): 905-915. DOI: http://dx.doi.org/10.26418/jhl.v7i2. 34559

Samoisy, A.K. \& Mahomodally, M.F. (2015). Ethnopharmacological Analysis of Medicinal Plants Used Against NonCommunicable Diseases in Rodrigues Island, Indian Ocean. Journal of Ethnopharmacology. 173: 20-38. http://dx.doi.org/10.1016/j.jep.2015.06.03 6.

Taibi, K., Abderrahim, L.A., Ferhat, K., Betta, S., Taibi, F., Bouraada, F. \& Boussaid, M (2020). Ethnopharmacological Study of Natural Products Used for Traditional Cancer Therapy in Algeria. Saudi Pharmaceutical Journal, 28(11): 1451$1465 . \quad$ DOI: http://doi.org/10.1016/j.sjbs.2020.09.011.

Ullah, R., Alqahtani, A.S., Noman, O.M.A., Alqahtani, A.M., Ibenmoussa, S. \& Bourhia, M. (2020). A Review on ethno medicinal Plants Used in Traditional Medicine in The Kingdom of Saudi Arabia. Saudi Journal of Biological Science, 27(10): 2706-2718. DOI: http://doi.org/10.1016/j.sjbs.2020.06.020.

Yusro, F., Pranaka, R.N., Budiastutik. E. \& Mariani Y. (2020). Diversity of Medicinal Plants Used by Traditional Healers of Dayak Tribe in the Villages of Kebong and Merpak, Sintang Regency. Jurnal Biologi Tropis 20(3): 329-339. DOI: http://dx.doi.org/10.29303/jbt.v20i3.2010 